\title{
Performance Evaluation of Image Fusion Methods
}

\author{
Vassilis Tsagaris, Nikos Fragoulis and Christos Theoharatos \\ Irida Labs \\ Greece
}

\section{Introduction}

The recent advances in sensor technology, microelectronics and multisensor systems have motivated researchers towards processing techniques that combine the information obtained from different sensors. For this purpose a large number of image fusion techniques [Mukhopadhyay \& Chanda, 2001; Pohl \& van Genderen, 1998, Tsagaris \& Anastassopoulos, 2005; Piella, 2003] have been proposed in the fields of remote sensing, medical diagnostics, military applications, surveillance etc. The main goal of these image fusion techniques is to provide a compact representation of the multiple input images into a single grayscale one that contains all the important original features. Such an image provides improved interpretation capabilities but can also be used for further computer processing tasks, like feature extraction or classification.

The performance of image fusion techniques is sometimes assessed subjectively by human visual inspection. The reproduction of subjective tests is often time-consuming and expensive, while the exact same conditions for the test cannot be guaranteed. This has led to a rising demand for objective measures in order to rapidly compare the results obtained with different algorithms or to obtain optimal settings for a specific fusion algorithm. The objective evaluation of the performance of pixel level fusion methods is addressed in this book chapter. The image fusion processes can be classified in grayscale or color methods depending on the resulting fused image.

For this purpose the general framework of objective evaluation of image fusion is discussed and different fusion measures are discussed. Moreover, a global measure for grayscale image fusion schemes, IFPM, based on information theory is presented. The measure employs mutual and conditional mutual information in order to assess and represent the amount of information transferred from the source images to the final fused grayscale image. Accordingly, the common information contained in the source images is considered only once in the performance evaluation procedure. The experimental results clarify the applicability of the IFPM measure in comparing different fusion methods or in optimizing the parameters of a specific algorithm.

Moreover, a measure for objectively assessing the performance of color image fusion methods, CIFM, is presented in this chapter. Two different aspects are considered in establishing the measure, namely the amount of common information between the source images and the final fused image as well as the distribution of color information in the final 
image in order to achieve optimal color representation. Mutual information and conditional mutual information are employed in order to assess information transfer between the source images and the final fused image. Simultaneously, the distribution of colors in the final image is explored by means of the hue coordinate in the perceptually uniform CIELAB space. The proposed measure does not depend on the use of a target fused image for the objective performance evaluation. It is employed experimentally for objective evaluation of fusion methods in the cases of medical imaging and night vision data.

\section{Image fusion measures}

The problem of objective evaluation has not been addressed only in image fusion applications. A large number of metrics has been proposed over the years for assessing image and video fidelity. An informative overview on the topic can be found in [Avcibas et al, 2002]. These measures cannot be applied to evaluate image fusion methods since they require an ideal target image. Such an image is not always available as it happens in the field of remote sensing or medical imaging.

The performance of image fusion techniques is sometimes assessed subjectively by human visual inspection [Toet and Franken, 2003]. The reproduction of subjective tests is often time-consuming and expensive, while the exact same conditions for the test cannot be guaranteed. This has led to a rising demand for objective measures in order to rapidly compare the results obtained with different algorithms or to obtain optimal settings for a specific fusion algorithm.

In this context, [Xydeas \& Petrovic, 2000] proposed a measure based on edge information that is probably the first objective image fusion measure. The authors associated the important visual information with the "edge" information that is present in each pixel of an image. The evaluation of the amount of edge information that is transferred from input images to the fused image is employed as a measure of fusion performance. The edge detection process is based on Sobel algorithm that is applied both horizontally and vertically. The edge strength and the orientation information for each pixel are comprised and for an input image $\mathrm{A}$ we calculate

$$
\begin{gathered}
g_{A}(n, m)=\sqrt{S_{A}^{x}(n, m)^{2}+S_{A}^{y}(n, m)^{2}} \\
\alpha_{A}(n, m)=\arctan \left(\frac{S_{A}^{y}(n, m)}{S_{A}^{x}(n, m)}\right)
\end{gathered}
$$

Relative values of edge strength and orientation are calculated for a source image A and a fused image $\mathrm{F}$ is

$$
G^{A F}(n, m)=\left\{\begin{array}{cl}
\frac{g_{F}(n, m)}{g_{A}(n, m)} & \text { if } g_{A}(n, m) \geq g_{F}(n, m) \\
\frac{g_{A}(n, m)}{g_{F}(n, m)} & \text { otherwise }
\end{array}\right.
$$

These are used to derive the edge strength and orientation preservation values

$$
Q_{g}^{A F}(n, m)=\frac{\Gamma_{g}}{1+e^{k_{g}\left(G^{A F}(n, m)-\sigma_{g}\right)}}
$$




$$
Q_{\alpha}^{A F}(n, m)=\frac{\Gamma_{\alpha}}{1+e^{k \alpha\left(A^{A F}(n, m)-\sigma_{a}\right)}}
$$

$\mathrm{Q}_{\mathrm{g}}^{\mathrm{AF}}(\mathrm{n}, \mathrm{m})$ and $\mathrm{Q}_{\alpha}^{\mathrm{AF}}(\mathrm{n}, \mathrm{m})$ model perceptual loss of information in $\mathrm{F}$, in terms of how well the strength and orientation values of a pixel in A are represented in the fused image. The constants $\Gamma_{\mathrm{g}}, \mathrm{k}_{\mathrm{g}}, \sigma_{\mathrm{g}}$ and $\Gamma_{\mathrm{a}}, \mathrm{k}_{\mathrm{a}}, \sigma_{\mathrm{a}}$ determine the exact shape of the sigmoid functions used to form the edge strength and orientation preservation values. Edge information preservation values are then defined as

$$
Q^{A F}(n, m)=Q_{g}^{A F}(n, m) Q_{\alpha}^{A F}(n, m)
$$

while $\mathrm{Q}^{\mathrm{AF}}$ takes its values in the range zero to one. A value of zero corresponds to the complete loss of edge information, at location $(n, m)$, as transferred from $A$ into $F$. $Q^{\mathrm{AF}}=1$ indicates "fusion" from A to F with no loss of information. The authors have also proposed weighted versions of the $\mathrm{Q}^{\mathrm{AF}}$ criterion. The main drawback of this approach is the loss of information related with texture since it is mostly based on edge detection.

Moreover, in [Piella \& Heijmans, 2003], an image quality index proposed by [Wang \& Bovik, 2002] has been used for image fusion assessment. This measure is based on the second order statistics of both the source images and the final fused image, in order to assess fusion performance. The $\mathrm{Q}_{0}$ measure is calculated as:

$$
Q_{0}=\frac{4 \sigma_{x y} \bar{x} \bar{y}}{\left(\bar{x}^{2}+\bar{y}^{2}\right)\left(\sigma_{x}^{2}+\sigma_{y}^{2}\right)}
$$

but it can also be analyzed as:

$$
Q_{0}=\frac{\sigma_{x y}}{\sigma_{x} \sigma_{y}} \cdot \frac{2 \bar{x} \bar{y}}{\bar{x}^{2}+\bar{y}^{2}} \cdot \frac{2 \sigma_{x} \sigma_{y}}{\sigma_{x}^{2}+\sigma_{y}^{2}}
$$

Each image is a random variable $\mathrm{x}$, and its mean value and variance are $\overline{\mathrm{x}}, \sigma_{\mathrm{x}}^{2}$ respectively. The first term in (8) is the correlation coefficient between images $\mathrm{x}$ and $\mathrm{y}$. The value of $\mathrm{Q}_{0}$ ranges between -1 and 1 and is a measure of similarity between the two images. Piella and Heijmans, were based on the fact that image signals are generally non-stationary, thus it is more appropriate to measure the image quality index $Q_{0}$ over local regions and then combine the different results into a single measure. The fusion measure is given by

$$
Q(a, b, f)=\frac{1}{|w|} \sum_{w \in W}(\lambda(w)) Q_{0}(a, f \mid w)+(1-\lambda(w)) Q_{0}(b, f \mid w)
$$

where $\lambda(w)$ are local weights in the range of zero to one.

The ERGAS measure [Wald et al, 1997] which is an error index that offers a global picture of the quality of a fused product. The index is called ERGAS after its name in French, means relative dimensionless global error and is given by

$$
E R G A S=100 a \sqrt{\frac{1}{K} \sum_{k=1}^{K}\left(\frac{R M S E(k)}{\mu(k)}\right)^{2}}
$$

where a is the ratio between pixel sizes in cases of pansharpened and multispectral images, $\mu(\mathrm{k})$ is the mean of the $\mathrm{K}$ band and $\mathrm{K}$ is the number of bands. According to the authors, an ERGAS value greater than 3 corresponds to fused products of low quality, while an ERGAS value lower than 3 denotes a product of satisfactory quality. 
Especially for remote sensing applications, [Alparone et al, 2004] proposed the Q4 index that is suitable for MS imagery having four spectral bands. Both spectral and radiometric distortion measurements are encapsulated in a unique measurement, simultaneously accounting for local mean bias, changes in contrast, and loss of correlation of individual bands, together with spectral distortion.

More recently, the measures that have been proposed for objective evaluation of image fusion are based in information theory. These approaches provide a more general assessment of each image and have attracted the interest of a lot of researchers. A short discussion on basics of information theory is needed before presenting the IFPM and CIFM measures for grayscale and color image fusion respectively.

In [Qu et al, 2002], the authors introduced an information measure for image fusion assessment, which employs mutual information for representing the amount of information that is transferred from the source images to the final fused image. The overall fusion performance is the sum of mutual information between each source image and the final fused image. In this approach only the common information between each of the source images and the fused image is considered whereas no attention has been paid to the overlapping information of the source images. Additionally, the values of this measure are not bounded, e.g. in the range $[0-1]$, so the comparison between different fusion algorithms and data sets is not straightforward. The concepts of the overlapping information and comparable fusion performance are also considered in the following fusion measures.

\section{Information theory basics}

In this section the basic concepts from information theory, that are needed to describe the information and the common information between images, are provided. These concepts are used in different image fusion measures in order to evaluate and describe the amount of information in each image as well as common information between two or more images.

Each source image or the final grayscale image is considered as being a discrete random variable. The entropy or total information $\mathrm{H}(\mathrm{x})$ for a discrete random variable $\mathrm{X}$, is defined as

$$
H(X)=-\sum_{x} p(x) \log p(x)
$$

where $p(x)$ is the probability density function of the variable. Entropy is always a finite, positive number for discrete random variables and takes its maximum value in the case of a uniformly distributed variable. In the case of an image, entropy describes the total amount of information. The joint entropy $H(X, Y)$ for a pair of random variables $X, Y$ with joint distribution $p(x, y)$ is defined as

$$
H(X, Y)=-\sum_{x} \sum_{y} p(x, y) \log p(x, y)
$$

In addition the conditional entropy of a random variable $X$ given the random variable $Y$ is expressed as

$$
H(X \mid Y)=-\sum_{x} \sum_{y} p(x, y) \log p(x \mid y)
$$

The chain rule for two variables is expressed as $H(X, Y)=H(X)+H(Y \mid X)$. The generalized entropy chain rule is 


$$
H\left(X_{1}, X_{2}, \ldots, X_{N}\right)=H\left(X_{1}\right)+\sum_{i=2}^{N} H\left(X_{i} \mid X_{i-1}, \ldots, X_{1}\right)
$$

The common information shared between two random variables $X, Y$ is expressed by the mutual information that is defined as

$$
I(X ; Y)=\sum_{x} \sum_{y} p(x, y) \log \frac{p(x, y)}{p(x) p(y)}
$$

It can be proved that mutual information is always a positive quantity that vanishes only if $p(x, y)=p(x) p(y)$. Therefore, it can be interpreted as a measure of the statistical dependence between $X$ and $Y$. The relationship between mutual information and conditional entropy is given by

$$
I\left(X_{1}, X_{2}, \ldots, X_{N} ; Y\right)=H\left(X_{1}, X_{2}, \ldots, X_{N}\right)-H\left(X_{1}, X_{2}, \ldots, X_{N} \mid Y\right)
$$

The above quantities are schematically demonstrated by the Venn diagram of Figure 1.

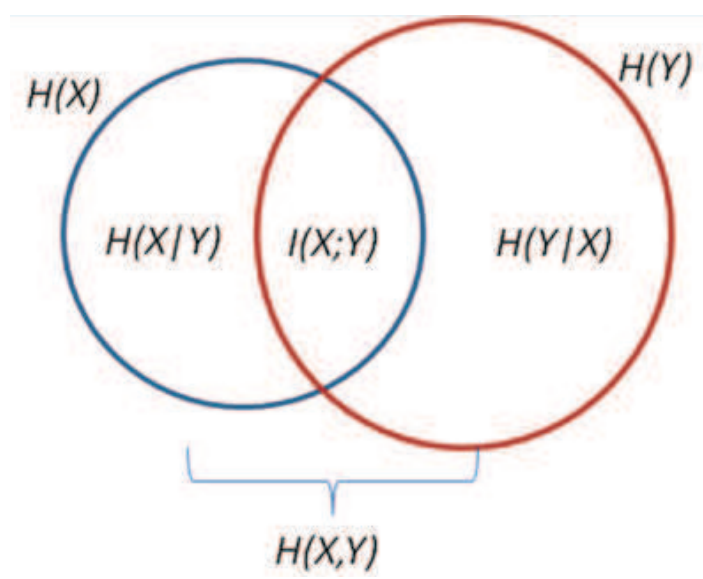

Fig. 1. The relationship between entropy and mutual information for two variables

The conditional mutual information of random variables $X_{1}$ and $X_{2}$ given $Y$ is defined by

$$
I\left(X_{1} ; Y \mid X_{2}\right)=H\left(X_{1} \mid X_{2}\right)-H\left(X_{1} \mid Y, X_{2}\right)
$$

and can be seen as the reduction in the uncertainty of $X_{1}$ due to the knowledge of $Y$ when $X_{2}$ is given [Cover \& Tomas, 1991]. The interpretation of conditional mutual information in a Venn diagram can be found in Figure 2. Apparently, conditional mutual information describes the shared information between two variables when a third variable has already been considered. Thus, conditional mutual information is employed to address the problem of overlapping information. The chain rule for mutual information is expressed as

$$
I\left(X_{1}, X_{2}, \ldots, X_{n} ; Y\right)=I\left(X_{1} ; Y\right)+\sum_{i=2}^{n} I\left(X_{1} ; Y \mid X_{i-1}, \ldots, X_{2}, X_{1}\right)
$$

The IFPM measure is based on mutual information in order to evaluate the amount of information that is transferred from the source images to the final fused representation. Moreover, the use of conditional mutual information guarantees that the overlapping information of the source images is considered only once. 

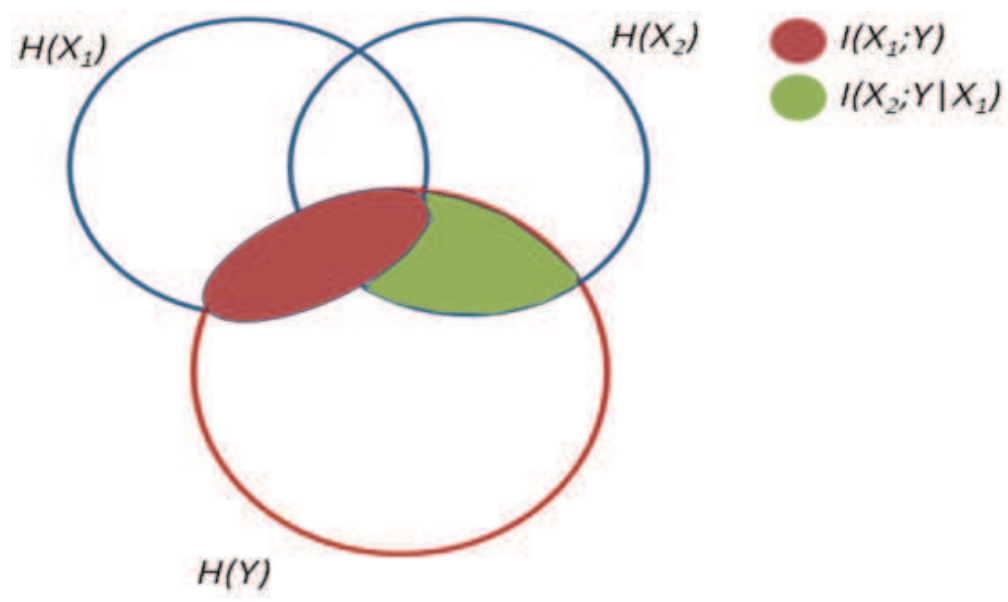

Fig. 2. The conditional mutual information for three variables

\section{Grayscale image fusion evaluation}

The purpose of an image fusion process is to combine a number of multimodal or multispectral images into a final entity that comprises the maximum possible information, which is present in the source images. The source images often exhibit a high degree of correlation since the same area is covered in different regions of the electromagnetic spectrum or with complementary imaging technologies. Thus, the same information can be found in more than one of the source images and is described as overlapping information. For example, in the case of multispectral imagery, each band reveals different aspects of the same scene but, at the same time, a large amount of overlapping information can be seen due to texture or spectral correlation. In an objective assessment of the effectiveness of a fusion algorithm the overlapping information should be considered only once and this problem has not been addressed in the existing measures.

\subsection{The IFPM measure}

The aim of the authors' work in [Tsagaris \& Anastassopoulos, 2006] was to provide an information-based global measure for objective performance evaluation of image fusion schemes. The proposed Image Fusion Performance Measure (IFPM) employs mutual information as well as conditional mutual information in order to evaluate the amount of information transferred from the source images to the final fused image.

IFPM is based on information quantities in order to objectively evaluate the performance of a fusion method. These quantities are evaluated all over the image resulting in a measure that can be regarded as global or universal. Each source image $X_{i}$ is treated as a discrete random variable with corresponding pdf $\left(x_{i}\right)$, while all the information quantities, described in the previous section, are employed. The resulting fused image is denoted as $Y$ while the corresponding probability density function as $p(y)$. Mutual information $I\left(X_{1} ; Y\right)$ describes the common information between the source image $X_{1}$ and the final fused image $Y$. The conditional mutual information $I\left(X_{2} ; Y \mid X_{1}\right)$ describes the common information between $X_{2}$ and $Y$ given $X_{1}$. In this way, only the information that is present in $X_{2}$ is considered in the evaluation of the common information between $X_{2}$ and $Y$. In its general form the conditional 
mutual information $I\left(X_{n} ; Y \mid X_{n-1}, \ldots, X_{2}, X_{1}\right)$ guarantees that the overlapping information between the source images $X_{i}$ is considered only once. The sum of all the conditional information represents the total amount of common information $C I$, transferred from the source images $X_{i}$ to the final fused image $Y$ and is expressed as

$$
C I=I\left(X_{1} ; Y\right)+I\left(X_{2} ; Y \mid X_{1}\right)+\cdots+I\left(X_{N} ; Y \mid X_{N-1}, \ldots, X_{1}\right)
$$

On the other hand the joint entropy $H\left(X_{1}, X_{2}, \ldots, X_{n}\right)$ represents the total amount of information that is present in the source images. The Image Fusion Performance Measure $(I F P M)$ is defined as

$$
I F P M=\frac{C I}{H\left(X_{1}, X_{2}, \ldots, X_{n}\right)}
$$

IFPM takes values in the range [0 - 1] where zero corresponds to total lack of common information between the source and the fused image and one corresponds to an effective fusion process that transfers all the information from the source images to the fused image (ideal case).

\subsection{Evaluation of grayscale image fusion based on IFPM}

In order to have a descriptive overview of objective evaluation we employ IF PM in order to compare four different fusion methods applied to three different data sets. The first fusion method that will be further referred to as Method 1, is a simple averaging of the source images. Method 2 is the well-known principal component analysis (PCA) algorithm, which is applied to the source images and the first principal component is considered as the final fused image. An approach based on discrete wavelet transform (DWT) and specifically on DBSS(2,2) is considered as Method 3 [Piella, 2003]. Finally, a fusion approach based on multiscale morphological pyramid [Mukhopadhyay and Chanda, 2001] is employed as Method 4.

The first data set used in this work consists of four multispectral bands and has been acquired by IKONOS-2 sensor. The radiometric resolution of each band is 11 bits. The ground resolution provided by IKONOS-2 for the multispectral imagery is $4 \mathrm{~m}$. The spectral range of the sensor is in the visible and near infrared region of the EM spectrum. The area covered in this multispectral image is mainly an urban area with a structured road network, a forest, a stadium, a park etc. The natural color composite image is shown in Figure 3(a), while in Figure 3(b), the near-infrared band is depicted. For perceptual comparison of the four fusion methods, their output fused images are demonstrated in Figure 3(c) to 3(f) for the fusion methods 1 to 4 respectively. A comparison of the fusion results at this point, from a perceptual point of view, reveals that all fusion methods provide an improved representation with methods 2 and 3 (PCA and DWT) to perform superiorly.

The second data set is derived from the night vision research area and comprises a color image of a scene representing a sandy path, trees and fences (Fig. 4(a)) and a midwave infrared $(3-5 \mu \mathrm{m})$ image in which a person is standing behind the trees and close to the fence, as shown in Fig. 4(b). The data set has been provided by TNO, Human Factors and a more detailed description of the data acquisition procedure can be found in [Toet, 2003]. The results of the four fusion methods are available for subjective visual evaluation in Figure 4(c)-4(f). Similar comments regarding the performance of the four methods, from a perceptual point, are valid for this data set as well. 


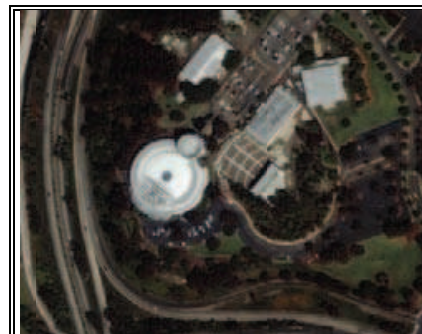

a)

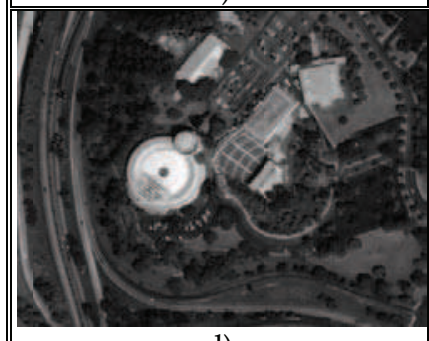

d)

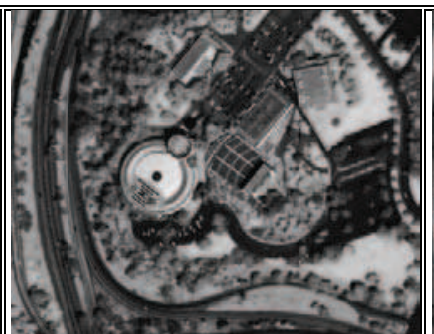

b)

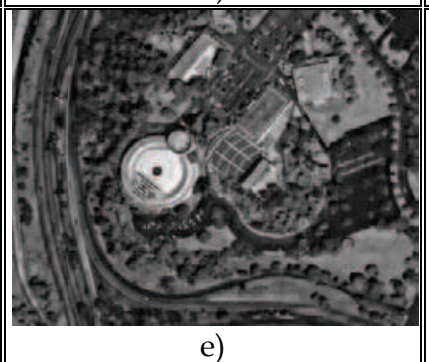

e)

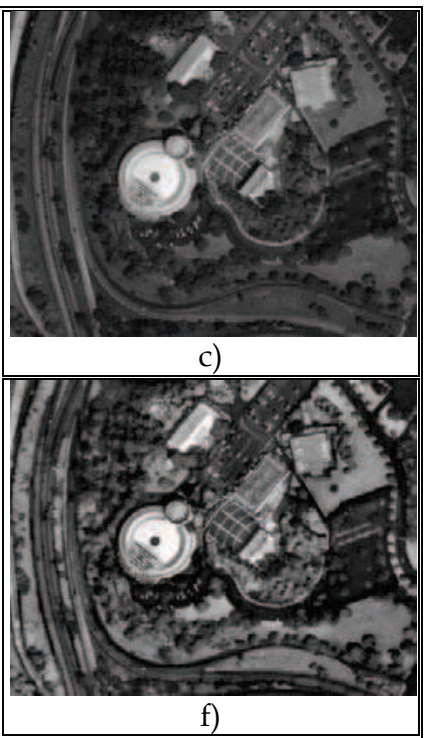

f)

Fig. 3. Source images (a) Natural color composite of the first three bands (b) the near infrared band. Results for (c) averaging (d) PCA (e) DWT and (f) morphological fusion methods

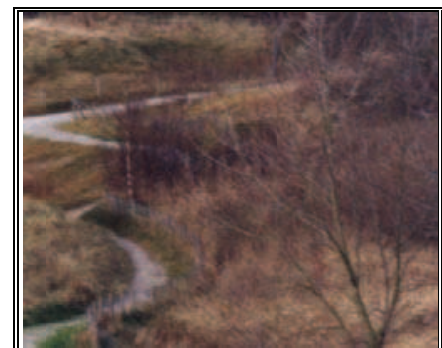

a)

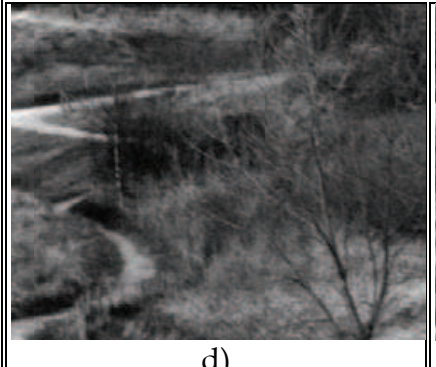

d)

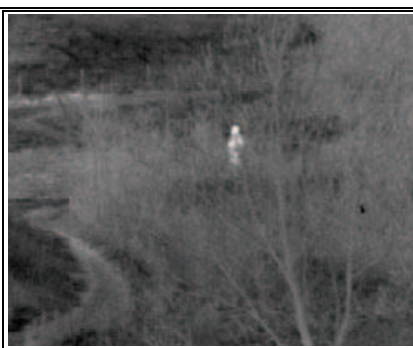

b)

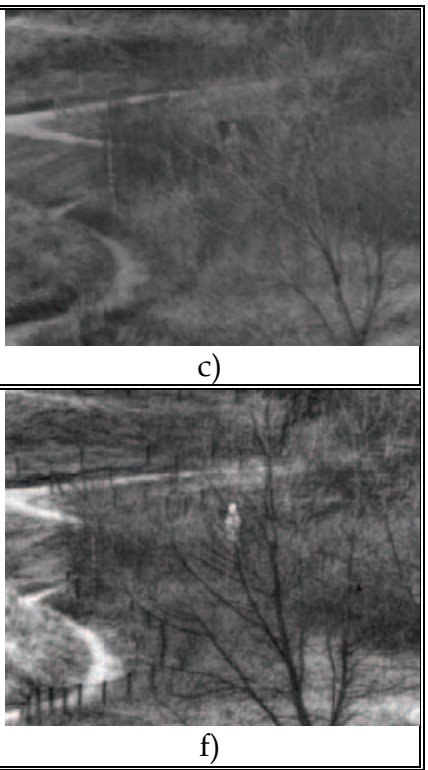

e)

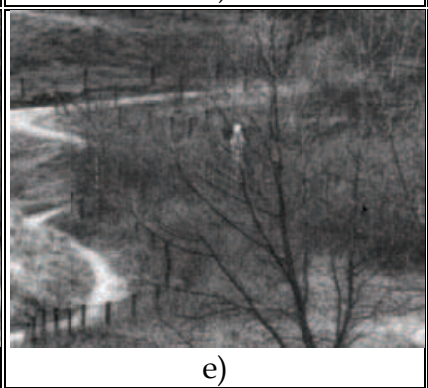

Fig. 4. Source images (a) Natural color composite of the first three bands (b) the near infrared band. Results for (c) averaging (d) PCA (e) DWT and (f) morphological fusion 
The evaluation of the four fusion methods for two different data sets based on the information theory measures can be found in Table 1 and reveals some interesting aspects of image fusion. The first conclusion is that there is no superior or outperforming image fusion method that can be used regardless the application. This conclusion has also been reported in [Toet and Franken, 2003, Qu et al, 2002] and proves that evaluation of image fusion performance in a real application, without having an ideal target image, is a complicated issue. Moreover, the type of the data involved in the image fusion process plays an important role. For example, in the case of high resolution multispectral data the source images to be fused possess a lot of features or objects with high edge information content. On the other hand, if thermal imagery is to be fused the visual features that are to be merged have a rather coarse outline.

The authors of [Tsagaris and Anastassopoulos, 2006] have compared the two measures in order to reveal the concept of overlapping information with a trivial case in which overlapping information is present between the source images. The results demonstrated that IFPM measure is not affected by the overlapping information, while the same conclusion does not hold for MI measure. Moreover, IFPM provide largest percentages of differentiation between the fusion methods and gives comparable results.

\begin{tabular}{|l|c|c|c|c|}
\hline & \multicolumn{2}{c|}{$\begin{array}{c}\text { Dataset 1 } \\
\text { Remote sensing }\end{array}$} & \multicolumn{2}{c|}{$\begin{array}{c}\text { Dataset 2 } \\
\text { Night vision }\end{array}$} \\
\hline & IFPM & MI & IFPM & MI \\
\hline Method 1 & 0.2629 & 3.4755 & 0.3104 & 2.0684 \\
\hline Method 2 & 0.2993 & 3.9023 & 0.3247 & 3.2043 \\
\hline Method 3 & 0.3050 & 3.4318 & 0.3704 & 2.8024 \\
\hline Method 4 & 0.2434 & 1.7036 & 0.4063 & 3.5632 \\
\hline
\end{tabular}

Table 1. Objective performance evaluation using IFPM and MI for the two datasets

\section{Color image fusion}

The objective measures discussed so far address the problem of grayscale image fusion that is fusion methods that result in grayscale representations. However, these measures cannot be trivially extended into color image fusion techniques.
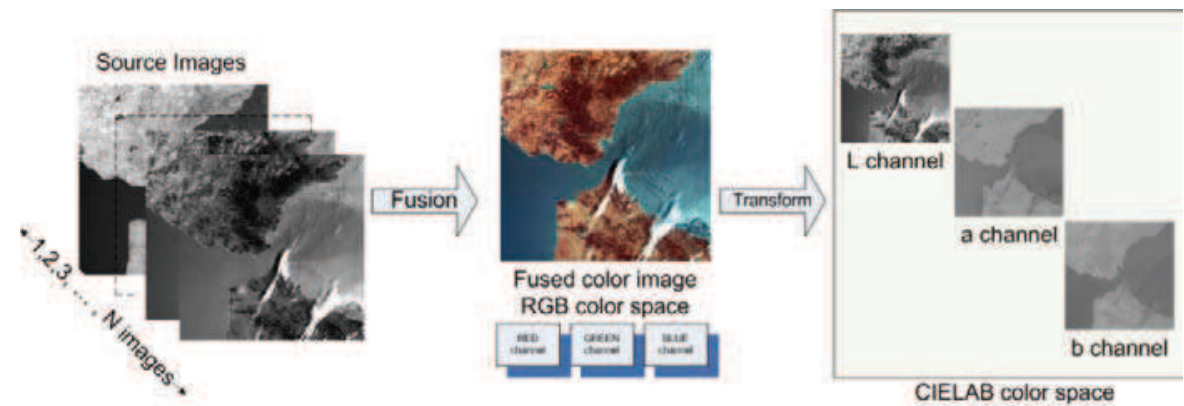

Fig. 5. Color image fusion process 
In an ordinary fusion procedure, $N$ multimodal or multispectral images are regarded as being the source images to be fused in order to produce a final color representation as shown in Figure 5. This color representation should perform as an ideal candidate for both detection and classification purposes and furthermore, be perceptually of high quality. Fusion methods that result in color images have to be assessed for the way they distribute the source information in the intensity, hue and saturation components since the main motivation behind color image fusion is the ability of the human vision system to distinguish thousands of colors. The fused color image is often formed in the RGB color space that is used in nowadays standard display devices and most computer vision tasks. However, the transformation of the color image in a uniform color space like CIELAB seems suitable in order to objectively evaluate the image fusion performance since in this color space the luminance component is independent of the chromaticity components.

Assessment of the effectiveness of a fusion method to result in a final image with maximum perceivable color information should be carried out in a perceptually uniform color space. The perceptually uniform CIELAB space consists of an achromatic luminosity component $L^{*}$ and two chromatic values $a^{*}$ and $b^{*}$, each one incorporating opponent colors [Malacara, 2002]. An alternative way to represent color characteristics is by transforming the $L^{*} a^{*} b^{*}$ components into cylindrical $C_{a b}^{*}$ and $h_{a b}^{*}$ coordinates on the $a^{*} b^{*}$ plane, given by

$$
\begin{gathered}
C_{a b}^{*}=\left[a^{* 2}+b^{* 2}\right]^{1 / 2} \\
h_{a b}^{*}=\arctan \left[\frac{b^{*}}{a^{*}}\right]
\end{gathered}
$$

where $C_{a b}^{*}$ correlates with chroma and $h_{a b}^{*}$ is an angle correlating with hue. In this system, an average observer is more sensitive to hue than to chroma differences [Malacara, 2002]. The IFPM measure employs the $h_{a b}^{*}$ coordinate to evaluate the color distribution in the final fused color image.

\subsection{The CIFM measure}

An approach for objectively assessing the performance of image fusion methods resulting in color images should address two issues. The problem of transferring information from the sources images and in the same time the problem of distributing this information in a color image that is color distribution in the final representation. The sources images could be either several grayscale images or a color image and grayscale images. In the case of a RGB color image each channel is regarded as a grayscale image.

The Color Image Fusion Measure (CIFM) presented in [Tsagaris, 2009] takes into consideration the amount of information transferred to the final image and, at the same time, the variety of colors obtained. It is a two component vector and each component deals with one of the two issues of the objective color image fusion evaluation previously described. The first terms concerns the amount of common information $C I$, between the intensity component of the final fused color image in the CIELAB color space, denoted as $L^{*}$ and the source images $X_{i}$ expressed as

$$
C I=I\left(X_{1} ; L^{*}\right)+I\left(X_{2} ; L^{*} \mid X_{1}\right)+\cdots+I\left(X_{N} ; L^{*} \mid X_{N-1}, \ldots, X_{1}\right)
$$

or equivalently

$$
C I=I\left(X_{1} ; L^{*}\right)+\sum_{i=2}^{N} I\left(X_{i} ; L^{*} \mid X_{i-1}, \ldots, X_{1}\right)
$$


The joint entropy $H\left(X_{1}, X_{2}, \ldots, X_{n}\right)$ represents the total amount of information that is present in the source images and is used in order to derive a normalized version of the amount of common information. The Image Fusion Performance Measure (IFPM) was defined as

$$
I F P M=\frac{C I}{H\left(X_{1}, X_{2}, \ldots, X_{N}\right)}
$$

The angular coordinate $h_{a b}^{*}$ is additionally employed in order to evaluate the distribution of colors and hues in the final image. In [Tsagaris \& Anastassopoulos, 2005(b)] the concept of an image with Maximum Realizable Color Information (MRCI) and uniformly distributed information in the CIELAB color space and thus maximum perceivable information was proposed. The evaluation of the marginal distribution is carried out numerically using the uniformly distributed vector population in the CIELAB space for the MRCI image and it turns out that these marginal probabilities are not uniform [Tsagaris \& Anastassopoulos, 2005(b)] mainly due to the non-cylindrical shape of the CIELAB color space. In order to evaluate the color distribution the authors employed the angular coordinate $h_{a b}^{*}$ and its marginal probability density function $p\left(h_{a b}^{*}\right)$ which is calculated using

$$
p\left(h_{a b}^{*}\right)=\iint_{L, C} p\left(L^{*}, C_{a b}^{*}, h_{a b}^{*}\right) d L^{*} d C_{a b}^{*}
$$

The color image resulting from the fusion process is considered in the CIELAB space and its $h_{a b}^{*}$ coordinate has a marginal pdf denoted as $q\left(h_{a b}^{*}\right)$. The Kullback-Leibler or relative entropy distance between the probability mass functions $p\left(h_{a b}^{*}\right)$ and $q\left(h_{a b}^{*}\right)$ is employed in order to quantify the similarity between the distribution of the color image resulted from the fusion process and the image with the maximum perceivable information. It is defined as

$$
D\left(p_{a b}^{*} \| q_{a b}^{*}\right)=\sum_{h} p\left(h_{a b}^{*}\right) h \log \frac{p\left(h_{a b}^{*}\right)}{q\left(h_{a b}^{*}\right)}
$$

If $q\left(h_{a b}^{*}\right)$ is close to $p\left(h_{a b}^{*}\right)$, the quantity $D\left(p_{a b}^{*} \| q_{a b}^{*}\right)$ is close to zero, which means that the image resulting from the fusion process, with histogram $q\left(h_{a b}^{*}\right)$ has a an almost ideal distribution of color and hues in the CIELAB space. In order to have an easily comparable measure we propose the Hue Distribution $(H D)$ is given by

$$
H D=1-D\left(p_{a b}^{*} \| q_{a b}^{*}\right)=1-\sum_{h} p\left(h_{a b}^{*}\right) h \log \frac{p\left(h_{a b}^{*}\right)}{q\left(h_{a b}^{*}\right)}
$$

The CIFM is expressed as

$$
C I F M=(I F P M, H D)
$$

The two vector components deals with the two problems of the objective color image fusion evaluation discussed in the previous section. A large value of IFPM indicates that a large amount of information is transferred from the source images to the luminance $L^{*}$ of the final fused color image. The use of mutual information along with conditional mutual information guarantees that no overlapping information is considered in this objective evaluation. Simultaneously, the $H D$ term measures the divergence of the hue coordinate in the CIELAB space of the fused color image from the hue coordinate of an image with uniform distribution in the CIELAB space. In this way, it provides an objective assessment of the variety of colors in the specially selected $h_{a b}^{*}$ coordinate. Both vector components are calculated in the CIELAB color space in order to take advantage of the perceptual 
uniformity of this color space and the independency between the luminosity component $L^{*}$ and the chromatic components.

\subsection{Objective evaluation of color image fusion methods}

The proposed CIFM is experimentally used in this section in order to assess the performance of four different fusion methods resulting in color images. In the following paragraphs a short description of the tested fusion methods is provided. Moreover, the experimental data from three different application areas are presented. Then, the two components of the CIFM measure, namely IFPM and HD are calculated for all data sets and for each fusion method and the results are discussed in order to derive conclusions.

Method 1 - The first fusion scheme, will be referred to as Method 1, is the well known Karhunen-Loewe transform or equivalently principal components analysis (PCA). In this approach the source data are transformed into an orthogonal space and then the first three principal components corresponding to the largest eigenvalues of the covariance matrix, are mapped to the RGB channels in order to form a color image.

Method 2 - This color image fusion scheme is based on perceptual attributes [Tsagaris \& Anastassopoulos, 2005]. The approach takes into consideration the inherent high correlation of the RGB bands in natural images. The resulting image is directly formed in the RGB color space and no further transformation is needed. The main advantage of the method is that it results in fused color images with adjustable covariance matrix.

Method 3 - A large variety of color image fusion methods based on wavelet methods has been proposed in the literature [Piella, 2003]. In these approaches the wavelet decomposition of the original images is merged using different fusion rules applied to the approximations coefficients and the details coefficients. Then inverse wavelet transform is applied to the merged coefficients in order to derive the final fused color image. Method 3 is based on the DBSS $(2,2)$ wavelet and fusion is applied by taking the maximum for approximation and the minimum for the details coefficients.

Method 4 - Finally, Method 4 [Tsagaris \& Anastassopoulos, 2005] is a fusion scheme based on non-negative matrix factorization [Lee \& Seung, 1999] and the application of a color transfer technique. In this way an additive representation of the source features is obtained while inappropriate color mappings are avoided due to the use of color transfer. Simultaneously, the overall discrimination capabilities in the final fused color image are enhanced.

The source experimental data employed in this work are three data sets one derived from the field of medical imaging, the second from the area of night vision and the third from the field of remote sensing. These data sets originate from different research fields and are acquired with different imaging techniques in order to cover in the experimental results different source data and a variety of research challenges in image fusion.

Dataset 1 - The first data set is composed of multi-modal medical images selected from the Brain Atlas collection of the Medical Harvard School. A four-dimensional vector space is considered, where a greyscale magnetic resonance image (MRI) and the three components of a computed tomography (CT) pseudo-color image, shown in Figure 6 (a) and (b) respectively, define the axes of this space. The two images are registered and of the same size. Employing the aforementioned fusion methods, four different final color images are obtained as shown in Figure 6(c) - (f) respectively.

Dataset 2 - The second data set is from the research field of night vision and thermal imaging, and was also described in the case of grayscale image fusion. It comprises a color 
image of a scene representing a sandy path, trees and fences and a midwave infrared (3$5 \mu \mathrm{m})$ image in which a person is standing behind the trees and close to the fence.

Dataset 3 - The third data set originates from the field of remote sensing. It consists of multispectral data acquired from the ENVISAT satellite and specifically MERIS sensor. MERIS (MEdium Resolution Imaging Spectrometer Instrument) measures the solar radiation reflected by the Earth at a ground spatial resolution of $300 \mathrm{~m}$, in 15 spectral bands, programmable in width and position, in the visible and near infra-red region of the electromagnetic spectrum. The geographical area is in the South part of Greece and covers both sea and land. The results of the previously described fusion methods can be found in Figure 7.

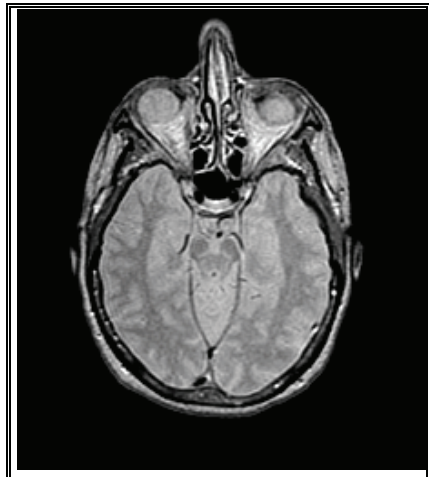

a)

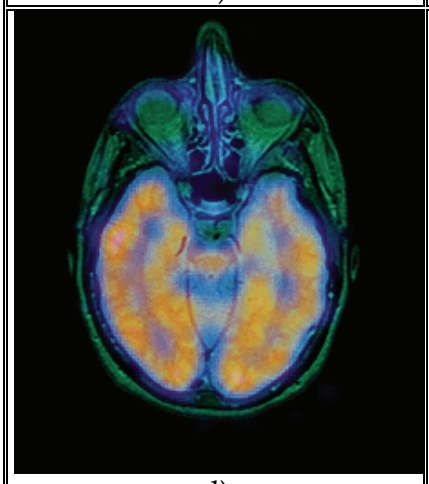

d)

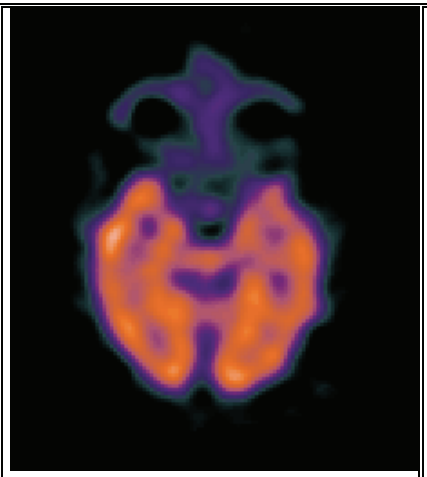

b)

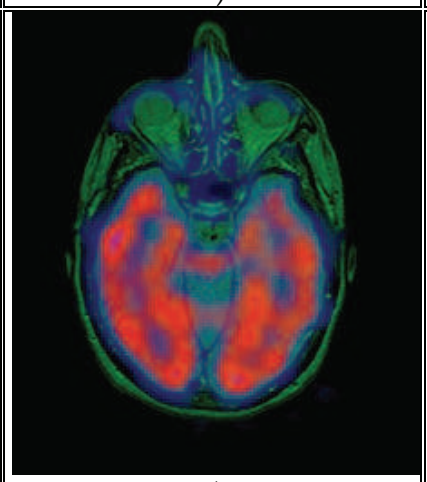

e)

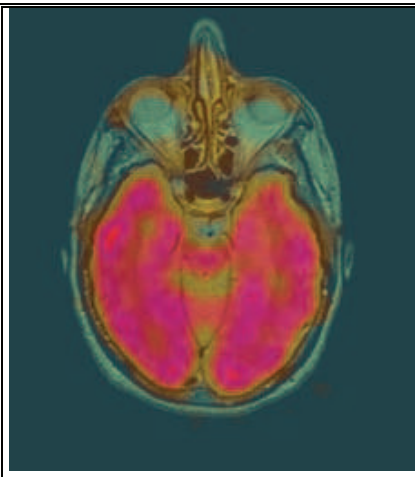

c)

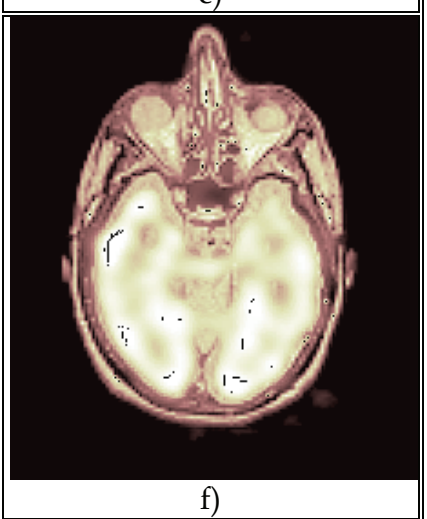

Fig. 6. Source data and fused color images. In (a) original MRI image and (b) the corresponding PET image (registered). (c) result of Method 1, (d) result of Method 2, (e) result of Method 3 and (f) result of Method 4

The proposed CIFM vector measure is calculated for the above described color image fusion methods. The two components of the measure namely, IFPM and HD are calculated and the results are summarized in Table 2 for the medical data. In the case of the medical data set Method 2 outperforms other methods for both IFPM and $H D$ vector components whilst 
Method 4, based on NMF is having a comparable performance. The first method based on PCA is having a significant performance as derived from the IFPM measure but it achieves poor results in the color distribution since the final color image is dominated by red color (first principal component). The Method 3 based on wavelet approach provides a good solution but not the best one.

The same procedure for the calculation of the proposed measure is applied for the case of the night vision data. In the case of the second dataset Method 3 provides the best results in both IFPM and HD measures. Method 2 is the second best solution and demonstrates a comparable high performance since it merges all the important features of the source images in the final fused representation. The poor performance of Method 1 is mainly due to the statistical nature of the PCA approach that fails to transfer small details of the source images in the final fused color image.

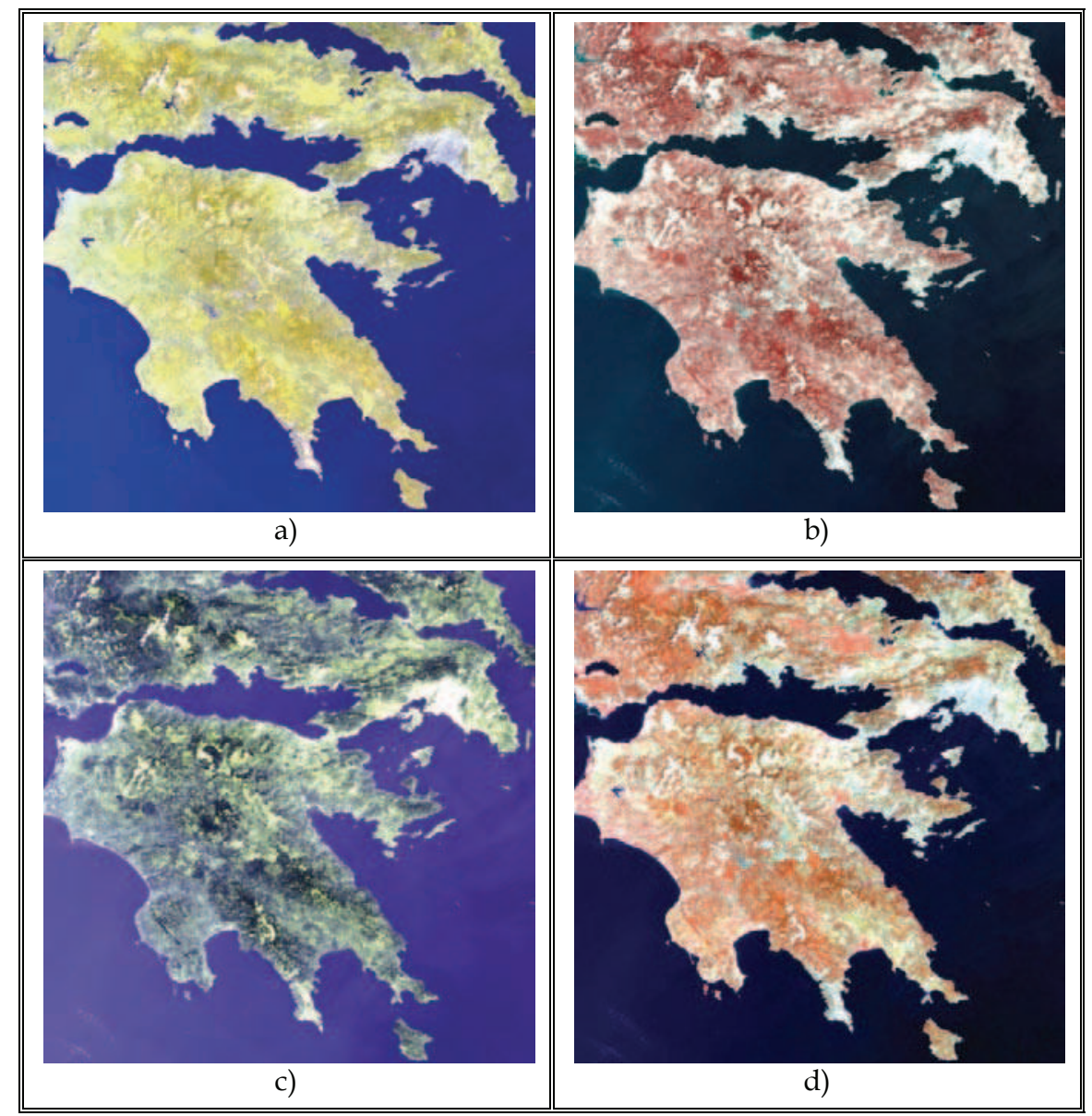

Fig. 7. Source Results of the fusion methods applied on MERIS data. In (a) result of Method 1, (b) result of Method 2, (c) result of Method 3 and (d) result of Method 4 
The vector components of the CIFM measure are also calculated for the case of the third dataset from the field of remote sensing. Method 2 achieves superior performance in both IFPM and HD components for the case of the third dataset. The fusion method that is based on the wavelet approach is having a comparable performance especially in the color distribution expressed by $H D$ measure. Method 4 achieves rather good but not optimal results in any measure whilst Method 1 fails to provide an efficient performance both in the information transfer but also in the color distribution.

These results are compliant with findings reported for the cases of grayscale image fusion [Toet \& Franken, 2003, Xydeas \& Petrovic, 2000, Qu et al, 2002, Tsagaris \& Anastassopoulos, 2006] which state that there is no superior image fusion method that can be applied in all datasets. Thus, objective evaluation of fusion schemes should be employed in order to decide on the best available solution for the specific application. Moreover, image fusion measures are also useful in parameter calculation and optimization for a specific image fusion method.

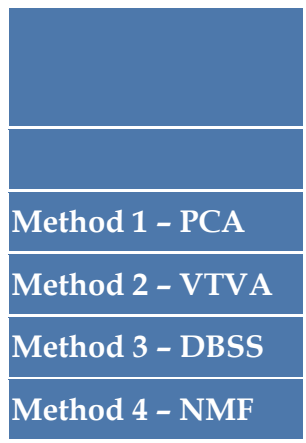

\begin{tabular}{|c|c|c|c|c|c|}
\hline \multicolumn{2}{c|}{$\begin{array}{c}\text { Dataset 1 } \\
\text { Medical data }\end{array}$} & \multicolumn{2}{c|}{$\begin{array}{c}\text { Dataset 2 } \\
\text { Night vision }\end{array}$} & \multicolumn{2}{c|}{$\begin{array}{c}\text { Dataset 3 } \\
\text { Remote sensing }\end{array}$} \\
\hline IFPM & $H D$ & IFPM & $H D$ & IFPM & $H D$ \\
\hline 0.3601 & 0.0503 & 0.1912 & 0.4848 & 0.2466 & 0.2811 \\
\hline 0.4850 & 0.9201 & 0.2423 & 0.6418 & 0.4248 & 0.7758 \\
\hline 0.3562 & 0.6216 & 0.2805 & 0.9034 & 0.3709 & 0.7558 \\
\hline 0.4485 & 0.8444 & 0.2085 & 0.5997 & 0.3687 & 0.5226 \\
\hline
\end{tabular}

Table 2. Objective performance evaluation using IFPM and $H D$ for the different datasets

The two components of the CIFM measure can also be used for a graphical representation in order to evaluate image fusion methods. The CIFM vector components, namely IFPM and $H D$ provide an orthogonal base for a two dimensional vector space (IFPM, HD) where each fusion method is regarded as a single point. Each vector component can also be used independently in certain applications. For example, IFPM could be employed if the amount of information transferred from the source images to the final image is important since further digital processing will be employed. On the other hand if the fused image will be used by visual experts then special attention should be given to the color distribution and thus $H D$ provide a useful tool.

The results of Table 2 are depicted in a graphical representation in Figure 8. The same conclusions about the performance of the four different fusion methods can be derived from this figure. Based on this representation, one may try to describe the CIFM measure in a scalar rather than a vector form, i.e. to use a distance expressed as $C I F M_{\text {alternative }}=$ $\sqrt{(1-I F P M)^{2}+(1-H D)^{2}}$. However, this or any similar approach fails to describe the two important issues in the color image fusion performance evaluation that is the assessment of information transferred to the final image and also the distribution of colors in the final fused color image and therefore should be employed in specific cases only. 
These results demonstrate that CIFM measure is a useful tool that can either replace or assist subjective evaluation. The experimental findings of the comparison of the four fusion methods prove that the proposed measure provides similar conclusions as those already known from the case of grayscale image fusion methods. That means there is no superior image fusion method that can be used independently of the application. Thus, objective performance evaluation based on CIFM should be employed in order to compare and choose between different image fusion methods for the specific application.

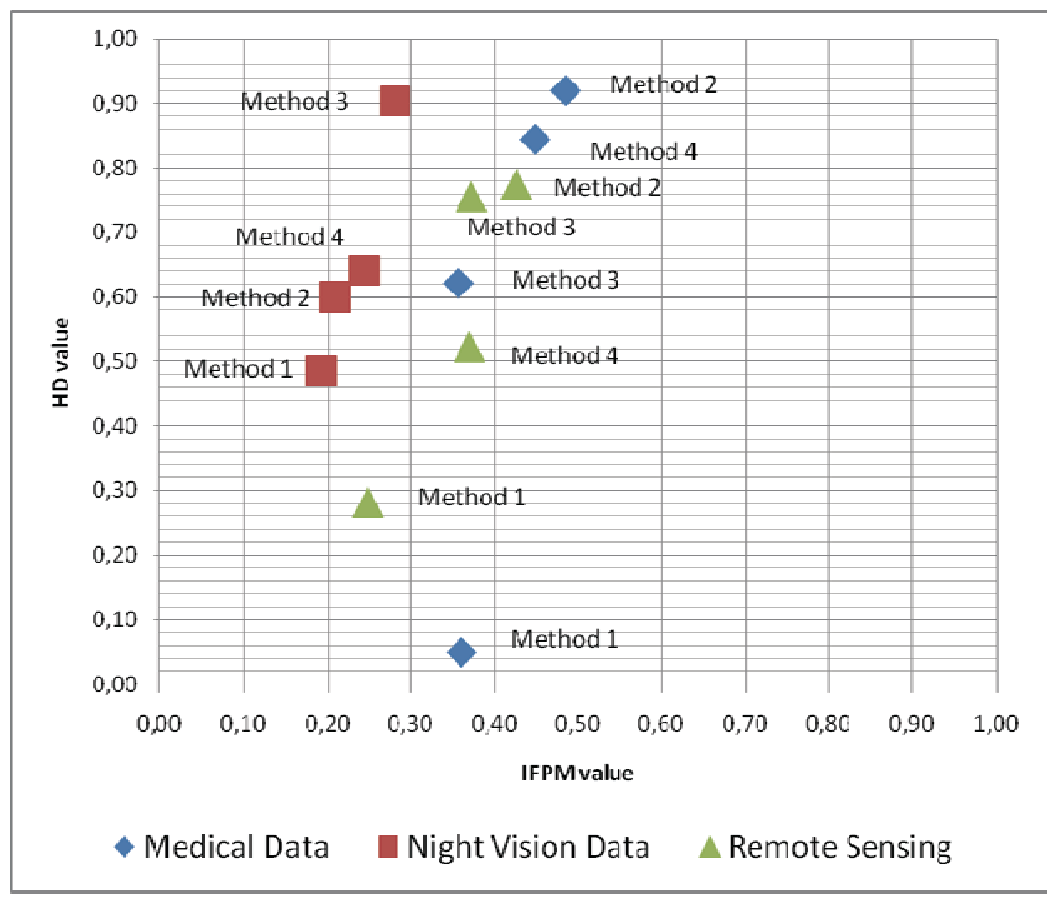

Fig. 8. Vector representation of image fusion performance for the different fusion methods and the three different data sets

\section{Future research}

The objective evaluation of image fusion methods is a challenging research problem that has attracted a lot of attention due to the new image fusion algorithms that are developed. The measures proposed up to now provide reliable objective evaluation based on information theory or other approaches. However, a thorough comparison of image fusion measures with subjective tests that can be easily reproduced would be very interesting.

Additionally, it is expected that in the near future the problem of dealing with regions instead of pixels will also affect the objective evaluation of image fusion methods. 
Simultaneously, the evolution in color image fusion methods will result in new objective measures for these applications. Finally, the use of objective evaluation in real time applications leads to a new research field dealing with the development of dedicated software/hardware for real time objective evaluation of image fusion.

\section{References}

Alparone L., Baronti S., Garzelli A. and Nencini F., A global quality measurement of Pansharpened multispectral imagery, IEEE Geoscience and Remote Sensing Letters, vol. 1, issue 4, pp. 313-317, 2004.

Avcibas A., Sankur B. and Sayood K., Statistical evaluation of image quality measures, Journal of Electronic Imaging, vol. 11, no. 2, pp. 206-223, 2002.

Cover T. M. and Thomas J.A., Elements of information theory, Wiley Series, 1991.

Lee D.D. and Seung H.S., Learning the parts of an object by non-negative matrix factorization, Nature, vol. 401, pp. 788-791, 1999.

Malacara D., Color vision and colorimetry: theory and applications, SPIE Press, Washington 2002.

Mukhopadhyay S., Chanda B., Fusion of 2D Grayscale Images Using Multiscale Morphology, Pattern Recognition, vol. 34, no. 10, pp. 1939-1949, 2001.

Piella G., A general framework for multiresolution image fusion: from pixels to regions, Information Fusion, vol. 4, pp. 259-280, 2003.

Piella G. and Heijmans H., A new quality metric for image fusion, Proceeding of the IEEE International Conference in Image Processing, ICIP-2003, vol. 3, pp. 173-176, 2003.

Pohl C., van Genderen J.L., Multisensor image fusion in remote sensing: concepts, methods and applications, Int. Journal of Remote Sensing, vol. 19, no.5, pp. 823-845, 1998.

Qu G., Zhang D. and Yan P., Information measure for performance of image fusion, Electronics Letters, vol. 38, pp. 313-315, 2002.

Toet A., Natural color mapping for multiband night vision imagery, Information fusion, vol. 4, pp.155-166, 2003.

Toet A. and Franken E.M., Perceptual evaluation of different image fusion schemes, Displays, vol. 24, pp. 25-37, 2003.

Tsagaris V. and Anastassopoulos V., Multispectral image fusion for improved RGB representation based on perceptual attributes, Int. Journal of Remote Sensing, vol. 26, no. 15, pp. 3241-3254, 2005.

Tsagaris V. and Anastassopoulos V., Assessing information content in color images, Journal of Electronic Imaging, vol 14, no, 4, 043007, 2005.

Tsagaris V. and Anastassopoulos V., Fusion of visible and infrared imagery for night color vision, Displays Journal, vol. 26, no. 4-5, pp. 191-196, 2005.

Tsagaris V. and Anastassopoulos V., A Global Measure for Assessing Image Fusion Methods, Optical Engineering vol. 45, no2, 2006 DOI: 10.1117/12.683964.

Tsagaris V., Objective evaluation of color image fusion methods, Optical Engineering vol. 48, 066201, 2009 DOI:10.1117/1.3153331. 
Wald L., Ranchin T. and Mangolini M., Fusion of satellite images of different spatial resolutions: assessing the quality of resulting images, Photogrammetric Engineering and Remote Sensing, vol. 63, no. 6, pp. 691-699, 1997.

Wang Z., Bovik A.C., A universal image quality index, IEEE Signal Processing Letters, vol. 9, no. 3, pp. 81-84, 2002.

Xydeas C.S. and Petrovic V., Objective image fusion performance measure, Electronics Letters, vol. 36, pp. 308-309, 2000. 


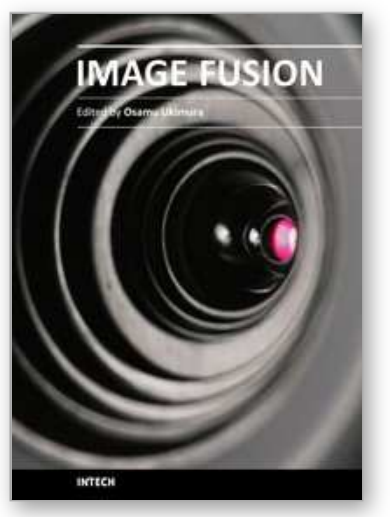

\author{
Image Fusion \\ Edited by Osamu Ukimura
}

ISBN 978-953-307-679-9

Hard cover, 428 pages

Publisher InTech

Published online 12, January, 2011

Published in print edition January, 2011

Image fusion technology has successfully contributed to various fields such as medical diagnosis and navigation, surveillance systems, remote sensing, digital cameras, military applications, computer vision, etc. Image fusion aims to generate a fused single image which contains more precise reliable visualization of the objects than any source image of them. This book presents various recent advances in research and development in the field of image fusion. It has been created through the diligence and creativity of some of the most accomplished experts in various fields.

\title{
How to reference
}

In order to correctly reference this scholarly work, feel free to copy and paste the following:

Vassilis Tsagaris, Nikos Fragoulis and Christos Theoharatos (2011). Performance Evaluation of Image Fusion Methods, Image Fusion, Osamu Ukimura (Ed.), ISBN: 978-953-307-679-9, InTech, Available from: http://www.intechopen.com/books/image-fusion/performance-evaluation-of-image-fusion-methods

\section{INTECH}

open science | open minds

\section{InTech Europe}

University Campus STeP Ri

Slavka Krautzeka 83/A

51000 Rijeka, Croatia

Phone: +385 (51) 770447

Fax: +385 (51) 686166

www.intechopen.com

\section{InTech China}

Unit 405, Office Block, Hotel Equatorial Shanghai

No.65, Yan An Road (West), Shanghai, 200040, China 中国上海市延安西路65号上海国际贵都大饭店办公楼405单元

Phone: +86-21-62489820

Fax: +86-21-62489821 
(C) 2011 The Author(s). Licensee IntechOpen. This chapter is distributed under the terms of the Creative Commons Attribution-NonCommercialShareAlike-3.0 License, which permits use, distribution and reproduction for non-commercial purposes, provided the original is properly cited and derivative works building on this content are distributed under the same license. 